\title{
On logarithmic Hölder continuity of mappings on the boundary
}

\author{
EVGENY SEVOST'YANOV
}

\begin{abstract}
We study mappings satisfying the so-called inverse Poletsky inequality. Under integrability of the corresponding majorant, it is proved that these mappings are logarithmic Hölder continuous in the neighborhood of the boundary points. In particular, the indicated properties hold for homeomorphisms whose inverse satisfy the weighted Poletsky inequality.
\end{abstract}

\section{Kuvausten logaritminen Hölderin jatkuvuus reunalla}

Tiivistelmä. Tutkimme kuvauksia, jotka toteuttavat ns. käänteisen Poletskyn epäyhtälön. Tilanteessa, jossa tähän liittyvä yläraja on integroituva, todistamme, että nämä kuvaukset ovat logaritmisesti Hölderin-jatkuvia reunapisteiden ympäristössä. Em. ominaisuudet pätevät erityisesti homeomorfismeille, joiden käänteiskuvaus toteuttaa painotetun Poletskyn epäyhtälön.

\section{Introduction}

As is known, quasiconformal mappings and mappings with bounded distortion are Hölder continuous with some exponent (see, e.g., [Ahl, Theorem III.C], [LV, Theorem 3.2.II], and [Va, Theorem 18.2, Remark 18.4]). In this regard, there are also classical results concerning mappings with bounded distortion, or quasiregular mappings, which are rightly called quasiconformal mappings with branch points (see, for example, $\left[\mathrm{MRV}_{1}\right.$, Theorem 3.2] and [Re, Theorem 1.1.2]). There are many generalizations of these results to more general classes of mappings with finite distortion, see e.g. [GGR, Theorem 1.1] and [GU, Theorem 5]. In this case, quite often, the usual estimates of the Hölder type do not hold for mappings, however, more general logarithmic estimates hold (see, for example, [Cr, Theorems 4 and 5], [MRSY Theorem 7.4], [MRSY ${ }_{2}$, Theorem 3.1], [RS, Theorem 5.11] and [Suv, Theorems 1.1.V and 2.1.V]).

Distance distortion theorems and Hölder-type estimates have been discussed and studied in our last two articles, [RSS] and [SSD]. In particular, in [SSD], we obtained estimates for the distortion of mappings with the inverse Poletsky inequality at the inner points of a given domain. The main purpose of this manuscript is to obtain similar estimates not only at the inner, but also at the boundary points of a given domain, which, for the sake of simplicity, will be assumed to be the unit ball.

In what follows, for the sets $A, B \subset \mathbb{R}^{n}$ we set, as usual,

$$
\operatorname{diam} A=\sup _{x, y \in A}|x-y|, \quad \operatorname{dist}(A, B)=\inf _{x \in A, y \in B}|x-y| .
$$

Let $x_{0} \in \bar{D}, x_{0} \neq \infty$,

$$
B\left(x_{0}, r\right)=\left\{x \in \mathbb{R}^{n}:\left|x-x_{0}\right|<r\right\}, \quad \mathbb{B}^{n}=B(0,1) .
$$

https://doi.org/10.54330/afm.113348

2020 Mathematics Subject Classification: Primary 30C65, 31A15, 30C62, 30C80.

Key words: Mappings with a finite and bounded distortion, Hölder continuous mappings, boundary behavior.

(c) 2022 The Finnish Mathematical Society 
Sometimes, instead of dist $(A, B)$, we also write $d(A, B)$, if a misunderstanding is impossible. A Borel function $\rho: \mathbb{R}^{n} \rightarrow[0, \infty]$ is called an admissible for a family $\Gamma$ of paths $\gamma$ in $\mathbb{R}^{n}$, if the relation

$$
\int_{\gamma} \rho(x)|d x| \geqslant 1
$$

holds for any locally rectifiable path $\gamma \in \Gamma$. A modulus of $\Gamma$ is defined as follows:

$$
M(\Gamma)=\inf _{\rho \in \operatorname{adm} \Gamma} \int_{\mathbb{R}^{n}} \rho^{n}(x) d m(x) .
$$

Let $Q: \mathbb{R}^{n} \rightarrow[0, \infty]$ be a Lebesgue measurable function. We say that $f$ satisfies the inverse Poletsky inequality, if the relation

$$
M(\Gamma) \leqslant \int_{f(D)} Q(y) \cdot \rho_{*}^{n}(y) d m(y)
$$

holds for any family of paths $\Gamma$ in $D$ and any $\rho_{*} \in \operatorname{adm} f(\Gamma)$. Note that estimates (1.3) hold in many classes of mappings (see, e.g., [MRV ${ }_{1}$, Theorem 3.2], [Ri, Theorem 6.7.II] and $\left[\mathrm{MRSY}_{1}\right.$, Theorem 8.5]). A mapping $f: D \rightarrow \mathbb{R}^{n}$ is called a discrete if $\left\{f^{-1}(y)\right\}$ consists of isolated points for any $y \in \mathbb{R}^{n}$, and an open, if the image of any open set $U \subset D$ is an open set in $\mathbb{R}^{n}$. A mapping $f$ between domains $D$ and $D^{\prime}$ is said to be a closed if $f(E)$ is closed in $D^{\prime}$ for any closed set $E \subset D$ (see, e.g., [ $\mathrm{Vu}_{1}$, Section 3]).

Given $\delta>0$, a non-degenerate continuum $A \subset \mathbb{B}^{n}$ and a Lebesgue measurable function $Q: \mathbb{B}^{n} \rightarrow[0, \infty]$ denote $\mathfrak{S}_{\delta, A, Q}$ a family of all open, discrete and closed mappings $f$ of the open unit ball onto itself such that the relation (1.3) holds and, in addition, $\operatorname{dist}\left(f^{-1}(A), \partial \mathbb{B}^{n}\right) \geqslant \delta$. The following statement holds.

Theorem 1.4. Let $Q \in L^{1}\left(\mathbb{B}^{n}\right)$. Then any $f \in \mathfrak{S}_{\delta, A, Q}$ has a continuous extension $f: \overline{\mathbb{B}^{n}} \rightarrow \overline{\mathbb{B}^{n}}$, and, in addition, for any $x_{0} \in \partial \mathbb{B}^{n}$ there is $C_{n}>0$ and $r_{0}=r_{0}\left(x_{0}\right)>0$ such that

$$
\left|f(x)-f\left(x_{0}\right)\right| \leqslant \frac{C_{n} \cdot\left(\|Q\|_{1}\right)^{1 / n}}{\log ^{1 / n}\left(1+\frac{r_{0}}{\left|x-x_{0}\right|}\right)}
$$

for $x \in B\left(x_{0}, r_{0}\right) \cap \overline{\mathbb{B}^{n}}$, where $\|Q\|_{1}$ denotes the $L^{1}$-norm of the function $Q$ in $\mathbb{B}^{n}$.

\section{Auxiliary lemma and proof of Theorem 1.4}

Before by proving the basic statement we prove the following important lemma.

Lemma 2.1. Let $E$ be a continuum in $\mathbb{B}^{n}$. Now, there is $\delta_{1}>0$ such that $\mathfrak{S}_{\delta, A, Q} \subset \mathfrak{S}_{\delta_{1}, E, Q}$. In other words, if $f$ is an open discrete and closed mapping of the unit ball onto itself with condition (1.3), such that $\operatorname{dist}\left(f^{-1}(A), \partial \mathbb{B}^{n}\right) \geqslant \delta$, then there is $\delta_{1}>0$, independent on $f$ such that $\operatorname{dist}\left(f^{-1}(E), \partial \mathbb{B}^{n}\right) \geqslant \delta_{1}$.

Proof. Let us prove this statement by contradiction. Suppose that the conclusion of the lemma is not correct. Then there are sequences $y_{m} \in E, f_{m} \subset \mathfrak{S}_{\delta, A, Q}$ and $x_{m} \in \mathbb{B}^{n}$ such that $f_{m}\left(x_{m}\right)=y_{m}$ and $\operatorname{dist}\left(x_{m}, \partial \mathbb{B}^{n}\right) \rightarrow 0$ as $m \rightarrow \infty$. Without loss of generality, we may assume that $x_{m} \rightarrow x_{0}$ as $m \rightarrow \infty$. By [SSD, Theorem 3.1] $f_{m}$ has a continuous extension to $x_{0}$, moreover, $\left\{f_{m}\right\}_{m=1}^{\infty}$ is equicontinuous at $x_{0}$ (see, e.g., [SSD, Theorem 1.2]). Now, for any $\varepsilon>0$ there is $m_{0} \in \mathbb{N}$ such that $\mid f_{m}\left(x_{m}\right)-$ $f_{m}\left(x_{0}\right) \mid<\varepsilon$ for $m \geqslant m_{0}$. On the other hand, $f_{m}\left(x_{0}\right) \in \mathbb{S}^{n-1}$ because $f_{m}$ is closed and hence the mapping $f$ preserves the boundary (see, for example, [ $\mathrm{Vu}_{1}$, Theorem 3.3]). Thus, by the triangle inequality, $\left|f_{m}\left(x_{m}\right)-f_{m}\left(x_{0}\right)\right| \geqslant 1-\left|f_{m}\left(x_{m}\right)\right| \geqslant 1-\delta_{0}$, where 
$\delta_{0}=\sup _{x \in E}|x|$. Finally, we have a contradiction, because $\left|f_{m}\left(x_{m}\right)-f_{m}\left(x_{0}\right)\right| \geqslant 1-\delta_{0}$ and, simultaneously $\left|f_{m}\left(x_{m}\right)-f_{m}\left(x_{0}\right)\right|<\varepsilon$ as $m \geqslant m_{0}$. The resulting contradiction refutes the assumption made above. Lemma is proved.

Proof of Theorem 1.4. Let $A=A\left(y_{0}, r_{1}, r_{2}\right)=\left\{y \in \mathbb{R}^{n}: r_{1}<\left|y-y_{0}\right|<r_{2}\right\}$ and let $\Gamma_{f}\left(y_{0}, r_{1}, r_{2}\right)$ denotes the family of all paths $\gamma:[a, b] \rightarrow D$ such that $f(\gamma) \in$ $\Gamma\left(S\left(y_{0}, r_{1}\right), S\left(y_{0}, r_{2}\right), A\left(y_{0}, r_{1}, r_{2}\right)\right)$, i.e., $f(\gamma(a)) \in S\left(y_{0}, r_{1}\right), f(\gamma(b)) \in S\left(y_{0}, r_{2}\right)$, and $\gamma(t) \in A\left(y_{0}, r_{1}, r_{2}\right)$ for $a<t<b$. Note that the mapping $f$ satisfies the condition

$$
M\left(\Gamma_{f}\left(y_{0}, r_{1}, r_{2}\right)\right) \leqslant \int_{A} Q(y) \cdot \eta^{n}\left(\left|y-y_{0}\right|\right) d m(y)
$$

for any $y_{0} \in \overline{\mathbb{B}^{n}}$ and any Lebesgue measurable function $\eta:\left(r_{1}, r_{2}\right) \rightarrow[0, \infty]$ such that

$$
\int_{r_{1}}^{r_{2}} \eta(r) d r \geqslant 1 .
$$

To establish this fact, it suffices to put $\rho_{*}(y):=\eta\left(\left|y-y_{0}\right|\right)$ for $y \in A \cap \mathbb{B}^{n}$ and $\rho_{*}(y)=0$ otherwise. By virtue of Luzin's theorem, we may assume that the function $\rho_{*}$ is Borel measurable (see, e.g., [Fe, Section 2.3.6]). Now, by [Va, Theorem 5.7] $\int_{\gamma_{*}} \rho_{*}(y)|d y| \geqslant$ $\int_{r_{1}}^{r_{2}} \eta(r) d r \geqslant 1$ for any (rectifiable) path $\gamma_{*} \in \Gamma\left(S\left(y_{0}, r_{1}\right), S\left(y_{0}, r_{2}\right), A\left(y_{0}, r_{1}, r_{2}\right)\right)$. Now, by (1.3), we obtain that

$$
M\left(\Gamma_{f}\left(y_{0}, r_{1}, r_{2}\right)\right) \leqslant \int_{\mathbb{B}^{n} \cap A} Q(y) \cdot \rho_{*}^{n}(y) d m(y)=\int_{\mathbb{B}^{n} \cap A} Q(y) \cdot \eta^{n}\left(\left|y-y_{0}\right|\right) d m(y),
$$

which was required to prove. Now, the possibility of a continuous extension of $f$ to the boundary of $\mathbb{B}^{n}$ is established in [SSD, Theorem 3.1]. In particular, the weakly flatness of $\partial \mathbb{B}^{n}=\mathbb{S}^{n-1}$ follows by [Va, Theorems 17.10 and 17.12].

Let us prove the logarithmic Hölder continuity (1.5). It suffices to prove relation (1.5) for the case when $x \in \mathbb{B}^{n} \cap B\left(x_{0}, r_{0}\right)$, since the general case $x \in \overline{\mathbb{B}^{n}} \cap B\left(x_{0}, r_{0}\right)$ is attained by passing to the limit as $x \rightarrow x_{*}$, where $x_{*} \in \partial \mathbb{B}^{n} \cap B\left(x_{0}, r_{0}\right)$. Let $x_{0} \in$ $\partial \mathbb{B}^{n}$, let $0<\delta<1$ and let $E=B(0, \delta / 2) \subset \mathbb{B}^{n}$. By Lemma 2.1, there is $\delta_{1}>0$ such that $\operatorname{dist}\left(f^{-1}(E), \partial \mathbb{B}^{n}\right) \geqslant \delta_{1}$ for any $f \in \mathfrak{S}_{\delta, A, Q}$. By [SSD, Theorem 1.2], the family $\mathfrak{S}_{\delta, A, Q}$ is equicontinuous in $\overline{\mathbb{B}^{n}}$. Thus, for a number $0<\delta_{0}<1 / 4$ there is a neighborhood $U \subset B\left(x_{0}, \delta_{1} / 2\right)$ of $x_{0}$ such that $|f(x)-f(y)|<\delta_{0}$ for any $x, y \in U \cap \mathbb{B}^{n}$ and $f \in \mathfrak{S}_{\delta, A, Q}$. Let $f(x) \neq f(y)$ and

$$
\varepsilon_{0}:=|f(x)-f(y)|<\delta_{0} .
$$

Let us join the points $f(x)$ and 0 by segment $I$. The points $f(x), 0$ and $f(y)$ form the plane $P$. Consider a circle

$$
S=\left\{z \in P:|z-f(x)|=\varepsilon_{0}\right\} .
$$

The position of the point $z=f(y)$ on the circle $S$ is completely determined by the angle $\varphi, \varphi \in[-\pi, \pi)$, between the vector $-f(x)$ and the radius-vector of the point $z$. The points on the circle are denoted further in the polar coordinates by the pairs $z=\left(\varepsilon_{0}, \varphi\right)$. Our further goal is to investigate the main three cases regarding the intervals of change of this angle.

Case 1. "Large angles": $\varphi \in[\pi / 4,3 \pi / 4]$, or $\varphi \in[-\pi / 4,-3 \pi / 4]$. Let $\varphi \in$ $[\pi / 4,3 \pi / 4]$. Consider the ray

$$
r=r(t)=f(y)+t e, \quad e=-f(x) /|f(x)|, \quad t>0 .
$$

By construction, the ray $r$ is parallel to the segment $I$. For $t=|f(x)|$, we have $r(|f(x)|)=f(y)-f(x)$ and $|r(|f(x)|)|=\varepsilon_{0}<\delta_{0}$, i.e., the point $r(|f(x)|)$ belongs to 
$E$. Let $J$ be a segment of the ray $r$, contained between the points $f(y)$ and $r(|f(x)|)$. The distance between $I$ and $J$ is calculated as follows:

$$
\operatorname{dist}(I, J)=\varepsilon_{0} \sin \varphi \geqslant \frac{\sqrt{2}}{2} \varepsilon_{0}
$$

see Figure 1 for illustration.

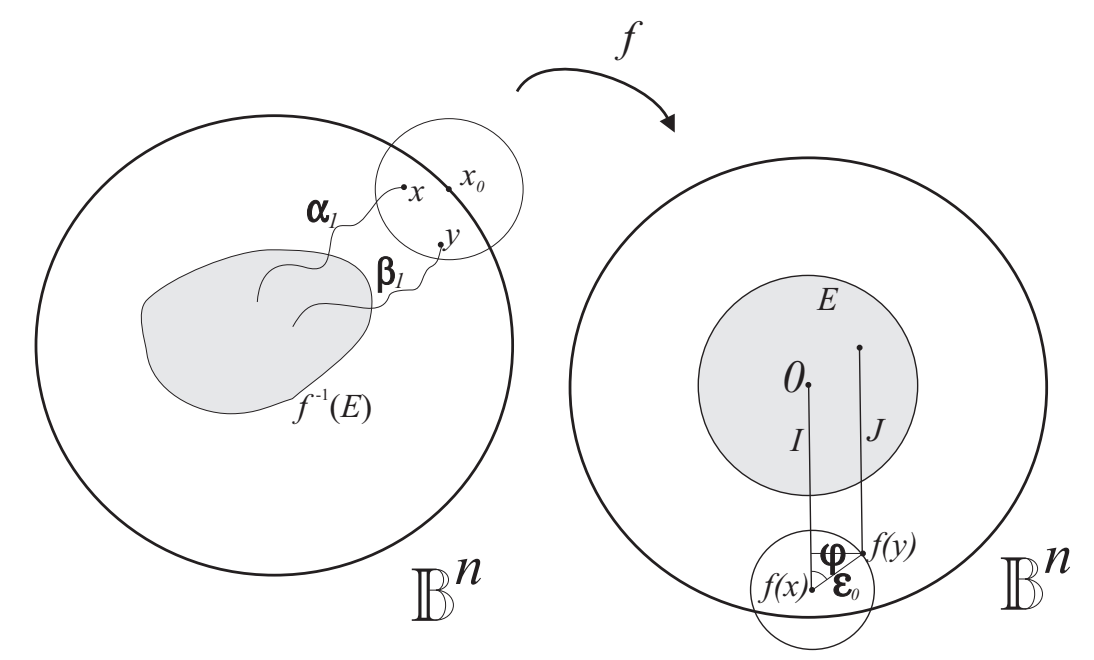

Figure 1. To the proof of Theorem 1.4, the case 1.

Similarly, the situation $\varphi \in[-\pi / 4,-3 \pi / 4]$ is considered. We may show that in this case the formula (2.3) also holds.

Let $\alpha_{1}$ and $\beta_{1}$ be (total) $f$-liftings of paths $I$ and $J$ starting at points $x$ and $y$, correspondingly. These liftings are well defined and exist by [ $\mathrm{Vu}_{1}$, Lemma 3.7]. Now, by the definition, $\alpha_{1} \cap f^{-1}(E) \neq \varnothing \neq \beta_{1} \cap f^{-1}(E)$. Since $\operatorname{dist}\left(f^{-1}(E), \partial \mathbb{B}^{n}\right) \geqslant \delta_{1}$ and $x, y \in B\left(x_{0}, \delta_{1} / 2\right)$,

$$
\operatorname{diam}\left(\alpha_{1}\right) \geqslant \delta_{1} / 2, \quad \operatorname{diam}\left(\beta_{1}\right) \geqslant \delta_{1} / 2 .
$$

Let

$$
\Gamma:=\Gamma\left(\alpha_{1}, \beta_{1}, \mathbb{B}^{n}\right) .
$$

Then on the one hand, by $\left[\mathrm{Vu}_{2}\right.$, Lemma 4.3]

$$
M(\Gamma) \geqslant(1 / 2) \cdot M\left(\Gamma\left(\alpha_{1}, \beta_{1}, \mathbb{R}^{n}\right)\right),
$$

and on the other hand, by [ $\mathrm{Vu}_{3}$, Lemma 7.38]

$$
M\left(\Gamma\left(\alpha_{1}, \beta_{1}, \mathbb{R}^{n}\right)\right) \geqslant c_{n} \cdot \log \left(1+\frac{1}{m}\right),
$$

where $c_{n}>0$ is come constant depending only on $n$,

$$
m=\frac{\operatorname{dist}\left(\alpha_{1}, \beta_{1}\right)}{\min \left\{\operatorname{diam}\left(\alpha_{1}\right), \operatorname{diam}\left(\beta_{1}\right)\right\}} .
$$

Combining (2.4) and (2.6), and taking into account that dist $\left(\alpha_{1}, \beta_{1}\right) \leqslant|x-y|$, we obtain that

$$
M(\Gamma) \geqslant \widetilde{c_{n}} \cdot \log \left(1+\frac{\delta_{1}}{2 \operatorname{dist}\left(\alpha_{1}, \beta_{1}\right)}\right) \geqslant \widetilde{c_{n}} \cdot \log \left(1+\frac{\delta_{1}}{2|x-y|}\right),
$$

where $\widetilde{c_{n}}>0$ is some constant depending only on $n$. 
We now obtain an upper bound for $M(\Gamma)$. Set

$$
\rho(x)= \begin{cases}\frac{\sqrt{2}}{\varepsilon_{0}}, & x \in \mathbb{B}^{n}, \\ 0, & x \notin \mathbb{B}^{n} .\end{cases}
$$

By (2.3) $\rho$ satisfies the relation (1.1) for the family $f(\Gamma)$. Then by the definition of the family $\mathfrak{S}_{\delta, A, Q}$ we obtain that

$$
M(\Gamma) \leqslant \frac{2^{n / 2}}{\varepsilon_{0}^{n}} \int_{\mathbb{B}^{n}} Q(y) d m(y)=2^{n / 2} \cdot \frac{\|Q\|_{1}}{|f(x)-f(y)|^{n}},
$$

where $\|Q\|_{1}$ denotes the $L^{1}$-norm of the function $Q$ in $\mathbb{B}^{n}$. By (2.7) and (2.8) we obtain that

$$
\widetilde{c_{n}} \cdot \log \left(1+\frac{\delta_{1}}{2|x-y|}\right) \leqslant 2^{n / 2} \cdot \frac{\|Q\|_{1}}{|f(x)-f(y)|^{n}} .
$$

The desired inequality (1.5) follows from the last relation by passing to the limit as $y \rightarrow x_{0}$, where $C_{n}:=2^{n / 2} \cdot{\widetilde{c_{n}}}^{-1 / n}$ and $r_{0}=d\left(x_{0}, \partial U\right)$.

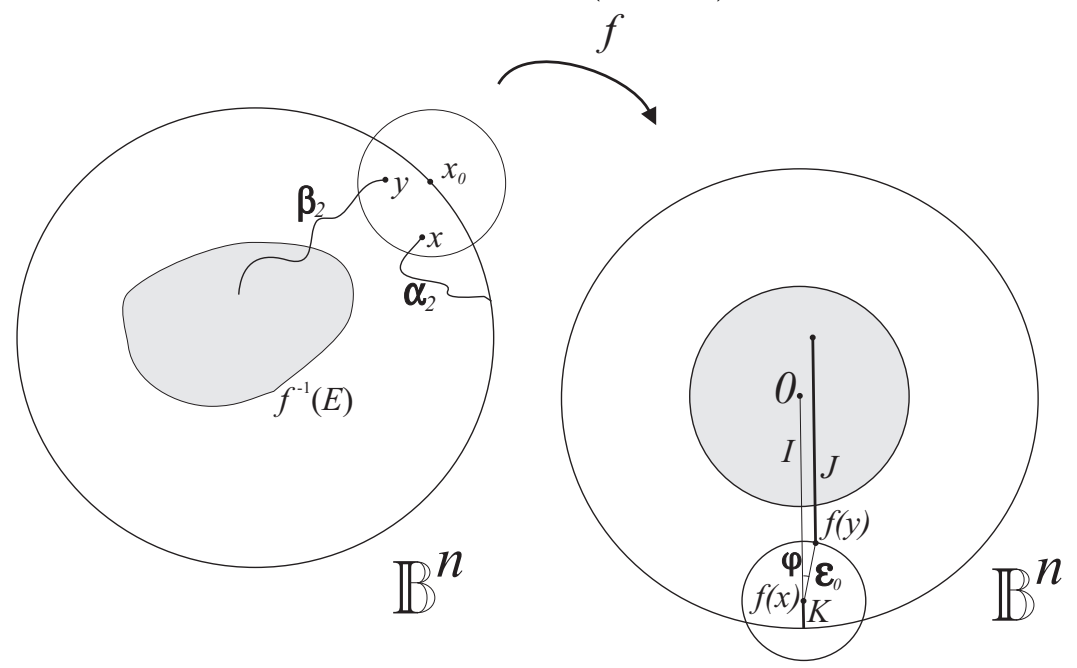

Figure 2. To the proof of Theorem 1.4, the case 2.

Case 2. "Small angles": $\varphi \in[-\pi / 4, \pi / 4)$, see Figure 2 for the illustration. In this case, we denote by $K$ the segment joining the point $f(x)$ with the unit sphere in the direction of the vector $f(x) /|f(x)|$. Then

$$
\operatorname{dist}(K, J)=\varepsilon_{0} .
$$

Let $\alpha_{2}$ be a maximal $f$-lifting of $K^{\prime}$ starting at $x$, where $K^{\prime}$ is obtained from $K$ by discarding its endpoint lying on the unit sphere. Such a lift exists and tends with its end to $\partial \mathbb{B}^{n}=\mathbb{S}^{n-1}$ (see, e.g., [MRV 2 , Lemma 3.12]). Let also $\beta_{2}$ be a total $f$-lifting of $J$ starting at the point $y$ (such a lifting exists by $\left[\mathrm{Vu}_{1}\right.$, Lemma 3.7]). Arguing similarly to a case 1 , we obtain that

$$
\operatorname{diam}\left(\beta_{2}\right) \geqslant \delta_{1} / 2 \text {. }
$$

Let

$$
\Gamma:=\Gamma\left(\alpha_{2} \cup \mathbb{S}^{n-1}, \beta_{2}, \overline{\mathbb{B}^{n}}\right) .
$$

According to the above, $\overline{\alpha_{2}} \backslash \alpha_{2} \subset \mathbb{S}^{n-1}$. Thus $\overline{\alpha_{2} \cup \mathbb{S}^{n-1}}=\overline{\alpha_{2}} \cup \mathbb{S}^{n-1}=\alpha_{2} \cup \mathbb{S}^{n-1}$, consequently, $\alpha_{2} \cup \mathbb{S}^{n-1}$ is a closed set. Observe that $\overline{\alpha_{2}}$ is connected (see, e.g., [Ku, Corollary 3(ii).II.46.5]). Moreover, the set $\alpha_{2} \cup \mathbb{S}^{n-1}=\overline{\alpha_{2}} \cup \mathbb{S}^{n-1}$ is connected as the union of two connected sets $\overline{\alpha_{2}}$ and $\mathbb{S}^{n-1}$, that have at least one common point (see, e.g., [Ku, Corollary 3(i).II.46.5]). 
In this case, on the one hand, by $\left[\mathrm{Vu}_{2}\right.$, Lemma 4.2]

$$
M(\Gamma) \geqslant(1 / 2) \cdot M\left(\Gamma\left(\alpha_{2} \cup \mathbb{S}^{n-1}, \beta_{2}, \mathbb{R}^{n}\right)\right),
$$

and on the other hand, by $\left[\mathrm{Vu}_{3}\right.$, Lemma 7.38]

$$
M\left(\Gamma\left(\alpha_{2} \cup \mathbb{S}^{n-1}, \beta_{2}, \mathbb{R}^{n}\right)\right) \geqslant c_{n} \cdot \log \left(1+\frac{1}{m}\right),
$$

where $c_{n}>0$ is some constant depending only on $n$,

$$
m=\frac{\operatorname{dist}\left(\alpha_{2} \cup \mathbb{S}^{n-1}, \beta_{2}\right)}{\min \left\{\operatorname{diam}\left(\alpha_{2} \cup \mathbb{S}^{n-1}\right), \operatorname{diam}\left(\beta_{2}\right)\right\}} .
$$

Then combining (2.10) and (2.12), and taking into account that $\operatorname{dist}\left(\alpha_{2} \cup \mathbb{S}^{n-1}, \beta_{2}\right) \leqslant$ $|x-y|$, we obtain that

$$
M(\Gamma) \geqslant \widetilde{c_{n}} \cdot \log \left(1+\frac{\delta_{1}}{2 \operatorname{dist}\left(\alpha_{2} \cup \mathbb{S}^{n-1}, \beta_{2}\right)}\right) \geqslant \widetilde{c_{n}} \cdot \log \left(1+\frac{\delta_{1}}{2|x-y|}\right),
$$

where $\widetilde{c_{n}}>0$ is some constant depending only on $n$.

Let us now establish an upper bound for $M(\Gamma)$. First of all, note that

$$
\Gamma\left(\alpha_{2} \cup \mathbb{S}^{n-1}, \beta_{2}, \overline{\mathbb{B}^{n}}\right) \supset \Gamma\left(\alpha_{2} \cup \mathbb{S}^{n-1}, \beta_{2}, \mathbb{B}^{n}\right)
$$

and

$$
\Gamma\left(\alpha_{2} \cup \mathbb{S}^{n-1}, \beta_{2}, \overline{\mathbb{B}^{n}}\right)>\Gamma\left(\alpha_{2} \cup \mathbb{S}^{n-1}, \beta_{2}, \mathbb{B}^{n}\right) .
$$

Therefore, by the principle of minority and in view of the monotonicity of the module we obtain that

$$
M\left(\Gamma\left(\alpha_{2} \cup \mathbb{S}^{n-1}, \beta_{2}, \overline{\mathbb{B}^{n}}\right)\right)=M\left(\Gamma\left(\alpha_{2} \cup \mathbb{S}^{n-1}, \beta_{2}, \mathbb{B}^{n}\right)\right)=M(\Gamma) .
$$

Set

$$
\rho(x)= \begin{cases}\frac{1}{\varepsilon_{0}}, & x \in \mathbb{B}^{n}, \\ 0, & x \notin \mathbb{B}^{n} .\end{cases}
$$

Observe that, by (2.9), $\rho$ satisfies the relation (1.1) for $f\left(\Gamma\left(\alpha_{2} \cup \mathbb{S}^{n-1}, \beta_{2}, \mathbb{B}^{n}\right)\right)$. In this case, by (2.14) and the definition of the family $\mathfrak{S}_{\delta, A, Q}$ we obtain that

$$
M(\Gamma)=M\left(\Gamma\left(\alpha_{2} \cup \mathbb{S}^{n-1}, \beta_{2}, \mathbb{B}^{n}\right)\right) \leqslant \frac{1}{\varepsilon_{0}^{n}} \int_{\mathbb{B}^{n}} Q(y) d m(y)=\frac{\|Q\|_{1}}{|f(x)-f(y)|^{n}} .
$$

By (2.13) and (2.15) we obtain that

$$
\widetilde{c_{n}} \cdot \log \left(1+\frac{\delta_{1}}{2|x-y|}\right) \leqslant \frac{\|Q\|_{1}}{|f(x)-f(y)|^{n}} .
$$

The desired inequality (1.5) follows from the last relation, which is achieved by passing to the limit as $y \rightarrow x_{0}$, where $C_{n}:={\widetilde{c_{n}}}^{-1 / n}$ and $r_{0}=d\left(x_{0}, \partial U\right)$.

Case 3. "Very large angles": either $\varphi \in(3 \pi / 4, \pi]$, or $\varphi \in(-\pi,-3 \pi / 4)$, see Figure 3 for the illustration. Let, for example, $\varphi \in(3 \pi / 4, \pi]$.

Consider on $P$ an auxiliary coordinate system centered at the point $z_{0}=0$, the abscissa axis of which we will position along the vector $f(x)$ with the direction opposite to this vector. The ordinate axis, respectively, we will position in the perpedicular direction so that the direction of this axis corresponds to the angle $\varphi=\pi / 2$ in the notation given above. Under these assumptions, each point $z=x+i y \in S, i^{2}=-1$, corresponds to its record in polar coordinates of the following form: $z=-|f(x)|+\varepsilon_{0} e^{i \varphi}, \varphi \in[-\pi, \pi)$. In this case,

$$
|z|^{2}=\left(-|f(x)|+\varepsilon_{0} \cos \varphi\right)^{2}+\varepsilon_{0}^{2} \sin ^{2} \varphi=|f(x)|^{2}-2 \varepsilon_{0}|f(x)| \cos \varphi+\varepsilon_{0}^{2} .
$$




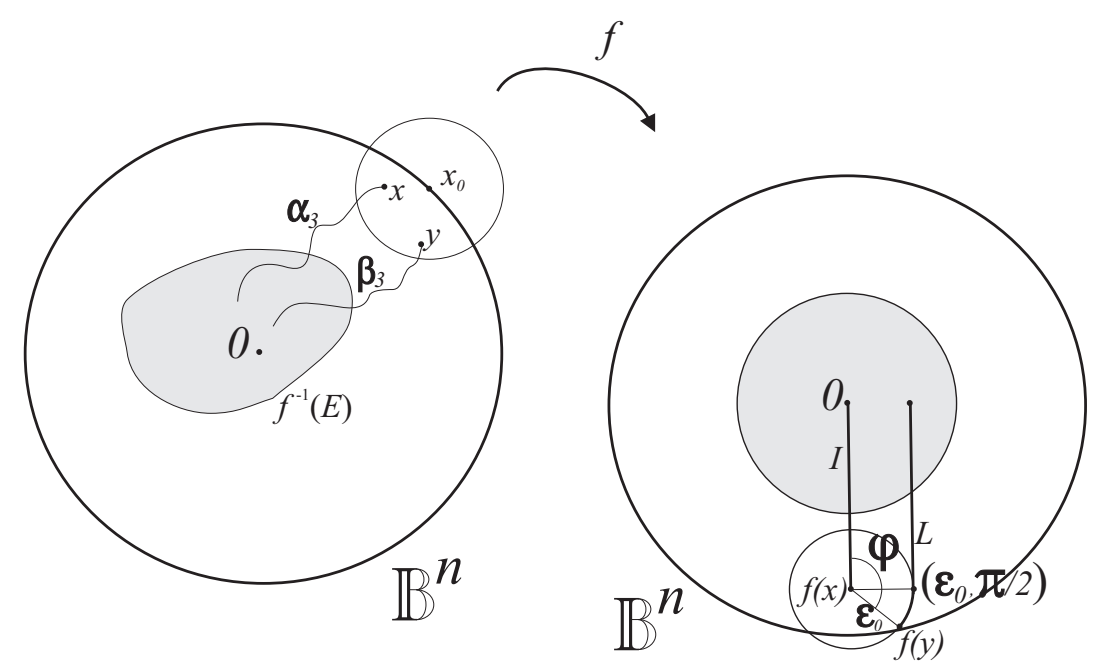

Figure 3. To the proof of Theorem 1.4, the case 3 .

It follows from $(2.16)$ that points $z=\left(\varepsilon_{0}, \varphi\right)$, having the largest absolute values of the number $\varphi, \varphi \in(3 \pi / 4, \pi]$, also have the largest Euclidean modulus of the number $z$. Thus, if $z=\left(\varepsilon_{0}, \varphi\right) \in \mathbb{B}^{n}$, then the whole arc of the circle

$$
z=z(\varphi)=\left\{\left(\varepsilon_{0}, \theta\right): \theta \in[\pi / 2, \varphi]\right\}
$$

also belongs to $\mathbb{B}^{n}$. Let us denote by $L_{1}$ the line segment

$$
l=l(t)=\left(\varepsilon_{0}, \pi / 2\right)+t e, \quad e=-f(x) /|f(x)|,
$$

corresponding to the values of the parameter $t \in[0,|f(x)|]$. Now, we obtain that $l(|f(x)|)=\left(\varepsilon_{0}, \pi / 2\right)-f(x)$, while $|l(|f(x)|)|=\varepsilon_{0}$ by the construction. Then $L_{1}$ intersects the ball $E$. Set $L=z(\varphi) \cup L_{1}$. We obtain that

$$
\operatorname{dist}(L, I)=\varepsilon_{0} \text {. }
$$

It can be shown that relation (2.17) is also satisfied for $\varphi \in(-\pi,-3 \pi / 4)$. Let $\alpha_{3}$ and $\beta_{3}$ be $f$-liftings of $I$ and $L$ starting $x$ and $y$, correspondingly. As before, the existence of such liftings is due to $\left[\mathrm{Vu}_{1}\right.$, Lemma 3.7]. Set $\Gamma=\Gamma\left(\alpha_{3}, \beta_{3}, \mathbb{B}^{n}\right)$. Further reasoning is similar to what was carried out in case 1 , namely, the existence of continua $I$ and $L$ with distance $\varepsilon_{0}$ implies that

$$
M(\Gamma) \geqslant \widetilde{c_{n}} \cdot \log \left(1+\frac{\delta_{1}}{2 \operatorname{dist}\left(\alpha_{3}, \beta_{3}\right)}\right) \geqslant \widetilde{c_{n}} \cdot \log \left(1+\frac{\delta_{1}}{2|x-y|}\right)
$$

and

$$
M(\Gamma) \leqslant \frac{1}{\varepsilon_{0}^{n}} \int_{\mathbb{B}^{n}} Q(y) d m(y)=\frac{\|Q\|_{1}}{|f(x)-f(y)|^{n}} .
$$

Hence we obtain the inequality

$$
\widetilde{c_{n}} \cdot \log \left(1+\frac{\delta_{1}}{2|x-y|}\right) \leqslant \frac{\|Q\|_{1}}{|f(x)-f(y)|^{n}} .
$$

From the last inequality we obtain the statement of the theorem due to the passage to the limit as $y \rightarrow x_{0}$.

The analog of Theorem 1.4 is also valid for mappings with a fixed point of the unit ball. In order to formulate and prove the corresponding statement, we introduce the following definition. For elements $a, b \in \mathbb{B}^{n}$ and the Lebesgue measurable function $Q: \mathbb{B}^{n} \rightarrow[0, \infty]$ denote by $\mathfrak{F}_{a, b, Q}$ the family of all open discrete and closed mappings $f$ of the unit ball onto itself, such that $f(a)=b$. The following statement is true. 
Theorem 2.18. Let $Q \in L^{1}\left(\mathbb{B}^{n}\right)$. Then any mapping $f \in \mathfrak{S}_{\delta, A, Q}$ has a continuous extension $f: \overline{\mathbb{B}^{n}} \rightarrow \overline{\mathbb{B}^{n}}$, in addition, for any $x_{0} \in \partial \mathbb{B}^{n}$ there exist $C_{n}>0$ and $r_{0}=r_{0}\left(x_{0}\right)>0$ such that the relation (1.5) holds.

Proof. The possibility of a continuous extension of the mapping $f$ to $\mathbb{S}^{n-1}$ follows from Theorem 3.1 in [SSD]. We prove the logarithmic Hölder continuity of the family of extended mappings. Let $E=\overline{B(0,1 / 2)}$. The following two cases are possible:

$1)$ there exists $\delta>0$ such that $\operatorname{dist}\left(f^{-1}(E), \partial \mathbb{B}^{n}\right) \geqslant \delta$ for any $f \in \mathfrak{S}_{\delta, A, Q}$. In this case, the desired statement follows from Theorem 1.4;

2) there exist $f_{m} \in \mathfrak{S}_{\delta, A, Q}$ and $x_{m}, y_{m} \in \mathbb{B}^{n}, m=1,2, \ldots$, such that $f_{m}\left(x_{m}\right)=y_{m}$, $y_{m} \in E$ and dist $\left(x_{m}, \mathbb{S}^{n-1}\right) \rightarrow 0$ as $m \rightarrow \infty$. Then, arguing exactly as in the proof of Lemma 2.1, we come to the conclusion that the family of mappings $\mathfrak{S}_{\delta, A, Q}$ is not equicontinuous at least at one point $x_{0} \in \mathbb{S}^{n-1}$, which contradicts the assertion of Theorem 7.1 in [SSD].

Thus, a case 2) is impossible, and a case 1) gives the desired statement of the theorem.

In addition to our article, we will illustrate our results with some simple examples.

Example 2.19. Consider a family of plane mappings $f_{n}(z)=z^{n}, n=1,2, \ldots$, $z \in \mathbb{B}^{2}=\{z \in \mathbb{C}:|z|<1\}$. The mappings $f_{n}$ have a bounded distortion as smooth mappings whose dilatation is equal to one. So $f_{n}$ satisfy inequality $(1.3)$ for $Q(z)=$ $N\left(f_{n}, \mathbb{B}^{2}\right)$, where, as usual, $N$ is a multiplicity function determined by the ratios

$$
N\left(y, f, \mathbb{B}^{2}\right)=\text { card }\left\{z \in \mathbb{B}^{2}: f(z)=y\right\}, \quad N\left(f, \mathbb{B}^{2}\right)=\sup _{y \in \mathbb{C}} N\left(y, f, \mathbb{B}^{2}\right)
$$

(see, e.g., $\left[\mathrm{MRV}_{1}\right.$, Theorem 3.2] or [Ri, Theorem 6.7.II]). All mappings $f_{n}$ are discrete and open, in addition, preserve the boundary of the unit disk and, therefore, are closed (see, e.g., $\left[\mathrm{Vu}_{1}\right.$, Theorem 3.3]). The mappings $f_{n}$ also fix the point 0 , so they satisfy all the conditions of Theorem 2.18 except one: the existence of the integrable function $Q$ in (1.3) independent on $n$. As a result, the family $f_{n}$ is not equicontinuous at the boundary of the unit disk.

Example 2.20. A simple example of conformal automorphisms $f_{n}(z)=\frac{z-\frac{n-1}{n}}{1-z \frac{n-1}{n}}$ of the unit disk on itself, for which $Q(z) \equiv 1$ (see, e.g., $\left[\mathrm{MRV}_{1}\right.$, Theorem 3.2] or [Ri, Theorem 6.7.II]), shows that the violation of the condition $\operatorname{dist}\left(f^{-1}(A), \partial \mathbb{B}^{n}\right) \geqslant \delta$ in Theorem 1.4 is also an obstacle to performing the desired inequality (1.5) on the boundary of the unit disk. We observe that the family $f_{n}$ is not even equicontinuous, and thus more, logarithmically Hölder continuous at the boundary points of the unit disk.

\section{References}

[Ahl] Ahlfors, L.: Lectures on quasiconformal mappings. - Van Nostrand, New York, 1966.

[Cr] Cristea, M.: Open discrete mappings having local $A C L^{n}$ inverses. - Complex Var. Elliptic Equ. 55:1-3, 2010, 61-90.

[Fe] Federer, H.: Geometric measure theory. - Springer, Berlin etc., 1969.

[GGR] Gol'dshtein, V., L. Gurov, and A. Romanov: Homeomorphisms that induce monomorphisms of Sobolev spaces. - Israel J. Math. 91, 1995, 31-60.

[GU] Gol'dshtein, V., and A. Ukhlov: About homeomorphisms that induce composition operators on Sobolev spaces. - Complex Variables and Elliptic Equations 55:8-10, 2010, $833-845$. 
[MRSY ${ }_{1}$ ] Martio, O., V. Ryazanov, U. Srebro, and E. Yakubov: Moduli in modern mapping theory. - Springer Science + Business Media, LLC, New York, 2009.

[MrSY ${ }_{2}$ ] Martio, O., V. Ryazanov, U. Srebro, and E. Yakubov: On $Q$-homeomorphisms. Ann. Acad. Sci. Fenn. Math. 30:1, 2005, 49-69.

[Ku] Kuratowski, K.: Topology. Volume 2. - Academic Press, New York-London, 1968.

[LV] Lehto, O., and K. Virtanen: Quasiconformal mappings in the plane. - Springer, New York etc., 1973.

[MRV 1 MARTIO, O., S. RICKMAN, and J. VÄISÄLÄ: Distortion and singularities of quasiregular mappings. - Ann. Acad. Sci. Fenn. Ser. A I Math. 465, 1970, 1-13.

$\left[\mathrm{MRV}_{2}\right]$ Martio, O., S. RICKMan, and J. VÄIsÄLÄ: Topological and metric properties of quasiregular mappings. - Ann. Acad. Sci. Fenn. Ser. A I Math. 488, 1971, 1-31.

[Re] Reshetnyak, Yu. G.: Space mappings with bounded distortion. - Transl. Math. Monogr. 73, Amer. Math, Soc., 1989.

[RS] Ryazanov, V., and E. Sevost'yanov: Toward the theory of ring $Q$-homeomorphisms. - Israel J. Math. 168, 2008, 101-118.

[RSS] Ryazanov, V., R. Salimov, and E. Sevost'yanov: On the Hölder property of mappings in domains and on boundaries. - J. Math. Sci. 246:1, 2020, 60-74.

[SSD] Sevost'yanov, E. A., S. A. Skvortsov, and O. P. Dovhopiatyi: On nonhomeomorphic mappings with the inverse Poletsky inequality. - Ukr. Mat. Visnyk 17:3, 2020, 414436 (in Ukrainian); Engl. transl. in J. Math. Sci. 252:4, 2021, 541-557.

[Suv] Suvorov, G. D.: Generalized principle of length and area in mapping theory. - Naukova Dumka, Kiev, 1985.

[Ri] Rickman, S.: Quasiregular mappings. - Springer-Verlag, Berlin, 1993.

$\left[\mathrm{Vu}_{1}\right] \quad$ VuORINEN, M.: Exceptional sets and boundary behavior of quasiregular mappings in $n$-space. - Ann. Acad. Sci. Fenn. Ser. A I Math. Diss. 11, 1976, 1-44.

[ $\left.\mathrm{Vu}_{2}\right]$ Vuorinen, M.: On the existence of angular limits of $n$-dimensional quasiconformal mappings. - Ark. Mat. 18, 1980, 157-180.

[Vu $\left.\mathrm{Vu}_{3}\right]$ Vuorinen, M.: Conformal geometry and quasiregular mappings. - Lecture Notes in Math. 1319, Springer-Verlag, Berlin etc., 1988.

[Va] VÄISÄLÄ, J.: Lectures on $n$-dimensional quasiconformal mappings. - Lecture Notes in Math. 229, Springer-Verlag, Berlin etc., 1971.

Received 12 January $2021 \bullet$ Accepted 8 April $2021 \bullet$ Published online 4 January 2022

Evgeny Sevost'yanov

Zhytomyr Ivan Franko State University

40 Bol'shaya Berdichevskaya Str., 10008 Zhytomyr, Ukraine; and

Institute of Applied Mathematics and Mechanics of NAS of Ukraine

1 Dobrovol'skogo Str., 84100 Slavyansk, Ukraine

esevostyanov2009@gmail.com 